\title{
Reaction Kinetic Study on Alternating Copolymerization of Vinyl Monomers Complexed with Alkylaluminum Halide
}

\author{
Hiroshi YabuUCHI ${ }^{\dagger}$ Masaaki HirooKa,${ }^{\dagger \dagger}$ and Isao KaETSU* \\ * Faculty of Science and Technology, Kinki University, \\ Kowakae, 3-4-1, Higashi-Osaka 577, Japan
}

(Received April 19, 1995)

\begin{abstract}
The kinetic scheme of the alternating copolymerization of a donor monomer and an acceptor monomer complexed with an alkylaluminum halide was studied to investigate the reaction mechanism. In the alternating copolymerization of styrene (St) and methyl acrylate (MA) complexed with aluminum ethylsesquichloride $\left(\mathrm{AlEt}_{1.5} \mathrm{Cl}_{1.5}\right)$, the kinetic data were expressed by the following equation (1). $R_{p}=k_{0}[\mathrm{MA}]^{1.0}\left[\mathrm{AlEt}_{1.5} \mathrm{Cl}_{1.5}\right]^{1.0}[\mathrm{St}]^{0}$ (1) Based on eq 1 together with the experimental observations the reaction mechanism of the alternating copolymerization was discussed.
\end{abstract}

KEY WORDS Alternating Copolymerization / Alkylaluminum Halide / Peroxide / Complex Formation / Methyl Acrylate / Styrene / Reaction Kinetics /

The alternating copolymerization of vinyl monomers having a low $e$-value in PriceAlfrey's $Q-e$ scheme (donor monomers) and conjugated vinyl monomers having a nitrile or carbonyl group (accepter monomer) complexed with an alkylaluminum halide has been extensively studied. ${ }^{1-5}$ It has been made possible to produce wide varieties of alternating copolymers with this method. They are not limited to some conventional alternating copolymers containing specific monomers such as maleic anhydride and its derivatives.

The reaction initiates spontaneously in the absence of any catalytic substances under the inert atomosphere such as nitrogen. In addition, it was observed that the reaction was promoted in the presence of benzoyl peroxide and oxygen. ${ }^{6}$ Some other characteristic reaction features have also been reported. ${ }^{7}$ But the reaction mechanism of the alternating copo- lymerization has not been fully investigated.

One of the effective method for elucidating the reaction mechanism of the alternating copolymerization is to study the kinetic scheme as it is generally applied in many cases. The kinetic equations reported in the past ${ }^{5,6}$ were reexamined and the revised kinetic equations were obtained. Through the kinetic study of the reaction some speculations on the reaction mechanism were proposed.

\section{EXPERIMENTAL}

\section{Materials}

Styrene was purified by washing with $10 \%$ aqueous sodium hydroxide and water, then drying over calcium chloride under nitrogen. The monomer was distilled in vacuum and stored over calcium hydride.

Methyl acrylate (MA) was washed with an

* To whom correspondence should be addressed.

† Present address: Sumitomo Chemical Company, Ltd., 5-33, Kitahama 4-chome, Higashi-ku, Osaka 577, Japan.

${ }^{\dagger \dagger}$ Present address: Faculty of Information Science, University of Marketing and Distribution Sciences, 3-1, Gakuen-Nishimachi, Nishi-ku, Kobe 673, Japan. 
aqueous solution containing 5\% sodium hydroxide and $20 \%$ sodium chloride, then with $20 \%$ aqueous sodium chloride. After cooling to $-78^{\circ} \mathrm{C}$ and separating ice, the monomer was dried over calcium chloride under nitrogen. It was distilled in vacuum and stored over calcium hydride.

Toluene of commercial G.R grade was used after drying over silica gel.

Ethylaluminum sesquichloride, obtained from the Ethyl Corporation Ltd. was distilled under nitrogen atmosphere, diluted with purified toluene and stored in glass amples.

\section{Polymerization and Its Rate Measurement}

In a 200-ml four-necked flask equipped with a stirrer, a thermometer and a glass inlet tube, toluene and methyl acrylate were mixed under nitrogen atmosphere and cooled to $-78^{\circ} \mathrm{C}$. When a toluene solution of ethylaluminum sesquichloride was added to this, the mixture became a homogeneous pale-yellow solution. Then the solution was brought to $25^{\circ} \mathrm{C}$. Upon the addition of styrene, occasionally with certain amount of peroxide as specified, polymerization reaction started. At a certain lapse of time the reaction mixture was poured into a large amount of methanol and white solid copolymer was recovered. The copolymer was washed with methanol, dried in vacuum and weighed. Reaction rate was caluculated based on the polymer weight.

\section{RESULTS AND DISCUSSION}

The polymerization rate was expressed as a first order reaction in terms of methyl acrylate concentration [MA] irrespective of the complex concentration [MA- $\left.\mathrm{AlEt}_{1.5} \mathrm{Cl}_{1.5}\right]$ and the free MA concentration [ $\left.\mathrm{MA}_{\mathrm{free}}\right]$ as shown in Figure 1. Styrene concentration [St] did not affect the rate of copolymerization as shown in Figure 2. The reaction rate was propotional to the alkylaluminum concentration $\left[\mathrm{AlEt}_{1.5} \mathrm{Cl}_{1.5}\right]$ as shown in Figure 3. The apparent rate constant is defined as $1 / t \times 2.303 \log [\mathrm{MA}]_{0} /[\mathrm{MA}]$,

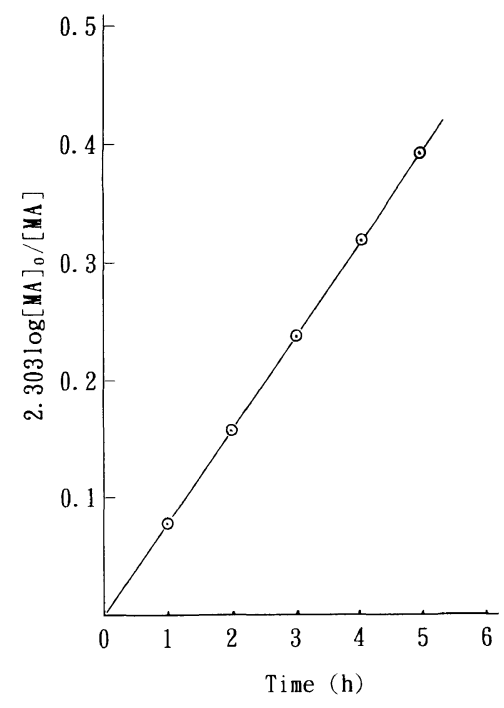

Figure 1. Time-conversion curves for the St-MA$\mathrm{AlEt}_{1.5} \mathrm{Cl}_{1.5}$ copolymerization at $25^{\circ} \mathrm{C}$. Solvent, toluene; $[\mathrm{MA}]_{0}=0.4 \mathrm{moll}^{-1} ;[\mathrm{St}]_{0}=0.6 \mathrm{moll}^{-1} ;\left[\mathrm{AlEt}_{1.5} \mathrm{Cl}_{1.5}\right]=$ $0.1 \mathrm{moll}^{-1}$.

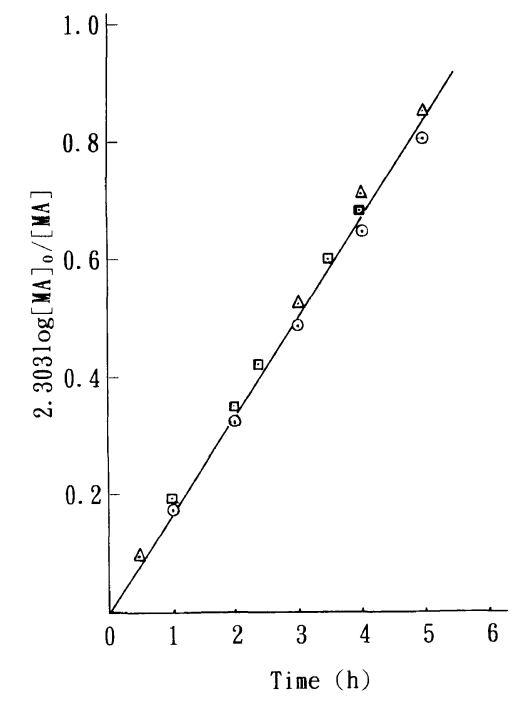

Figure 2. Effect of styrene concentration [St] on the rate of copolymerization at $25^{\circ} \mathrm{C}$. Solvent, toluene; $[\mathrm{MA}]_{0}=$ $0.4 \mathrm{moll}^{-1} ;\left[\mathrm{AlEt}_{1.5} \mathrm{Cl}_{1.5}\right]=0.2 \mathrm{moll}^{-1} ;[\mathrm{St}]_{0}:(\odot), 0.3$ $\mathrm{moll}^{-1} ;(\triangle), 0.6 \mathrm{moll}^{-1} ;(\bullet), 1.2 \mathrm{moll}^{-1}$.

$t=$ polymerization time. The overall kinetic data were therefore summarized by the following equation (1). 


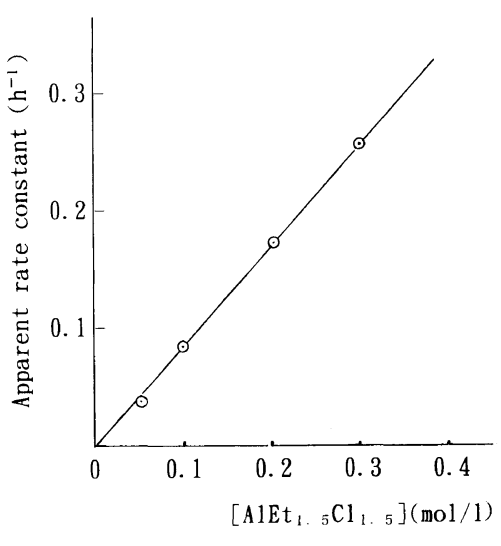

Figure 3. Effect of $\left[\mathrm{AlEt}_{1.5} \mathrm{Cl}_{1.5}\right]$ concentration on the rate of copolymerization at $25^{\circ} \mathrm{C}$. Solvent, toluene; $[\mathrm{MA}]_{0}=0.4 \mathrm{moll}^{-1} ;[\mathrm{St}]_{0}=0.6 \mathrm{moll}^{-1}$.

$$
\begin{aligned}
R_{\mathrm{p}} & =-\mathrm{d}[\mathrm{MA}] / \mathrm{d} t \\
& =k_{0}[\mathrm{MA}]^{1.0}\left[\mathrm{AlEt}_{1.5} \mathrm{Cl}_{1.5}\right]^{1.0}[\mathrm{St}]^{0}
\end{aligned}
$$

It was found in our experiments that the alkylaluminum compound remains unchanged in the course of the reaction without any consumption. So at a definite concentration of [AlEt ${ }_{1.5} \mathrm{Cl}_{1.5}$ ], eq 1 can be simplified by eq 2 as it is shown in Figure 1.

$$
R_{\mathrm{p}}=-\mathrm{d}[\mathrm{MA}] / \mathrm{d} t=k[\mathrm{MA}]^{1.0}
$$

Based on eq 1, speculations of the reaction mechanism could be proposed as follows although all of the speculations have not necessarily been proved by the experimental facts.

The complex formation and the propagation reaction of the alternating copolymerization might be assumed as follows.

$$
\mathrm{MA}+\mathrm{al} \rightleftarrows \mathrm{MA}-\mathrm{al}
$$

(I): Complex formation

$$
\sim \mathrm{St} \cdot+\mathrm{MA}-\mathrm{al} \rightarrow \sim \mathrm{St}-\mathrm{MA}(\mathrm{al}) \cdot
$$

$\sim \mathrm{St}-\mathrm{MA}(\mathrm{al}) \cdot \rightleftarrows \sim \mathrm{St}-\mathrm{MA} \cdot+\mathrm{al}$

(III): Regenaration of al

$\sim \mathrm{St}-\mathrm{MA} \cdot+\mathrm{St} \rightarrow \sim \mathrm{St} \cdot$

The polymerization rate $\left(R_{\mathrm{p}}\right)$ in terms of MA conversion could be expressed by the following manner in compliance with (II).

$$
R_{\mathrm{p}}=-\mathrm{d}[\mathrm{MA}] / \mathrm{d} t=k_{1}[\sim \mathrm{St} \cdot][\mathrm{MA}-\mathrm{al}]
$$

The complex formation (I) is considered to be a equiliblium reaction as it is generally known in many cases. ${ }^{8-10}$ The following (4) is the equation representing $K$ as an equiliblium constant.

$$
[\mathrm{MA}-\mathrm{al}]=K[\mathrm{MA}][\mathrm{al}]
$$

From eq 3 and $4, R_{\mathrm{p}}$ is expressed as follows.

$$
R_{\mathrm{p}}=k_{1} K[\mathrm{MA}][\mathrm{al}][\sim \mathrm{St} \cdot]
$$

$[\sim \mathrm{St} \cdot]$ could be determined by the stationary state assumption between the initiation rate $\left(R_{\mathrm{i}}\right)$ and the termination rate $\left(R_{\mathrm{t}}\right)$. The termination reaction might be either unimolecular termination (6) or bimolecular termination (7).

$$
\begin{aligned}
& R_{\mathrm{tu}}=k_{\mathrm{tu}}[\sim \mathrm{St} \cdot] \\
& R_{\mathrm{tb}}=k_{\mathrm{tb}}[\sim \mathrm{St} \cdot]^{2}
\end{aligned}
$$

If we take eq 7 and the stationary state assumption $\left(R_{\mathrm{i}}=R_{\mathrm{tb}}\right)$, eq 5 can be expressed as follows.

$$
R_{\mathrm{p}}=-\mathrm{d}[\mathrm{MA}] / \mathrm{d} t=k_{2}\left(\mathrm{Ri} / k_{\mathrm{tb}}\right)^{1 / 2}[\mathrm{MA}][\mathrm{al}]
$$

The eq 8 is basically identical with eq 1 in terms of [MA], [St], and [al] concentration, which was derived from the experimental measurement. The regeneration of alkylaluminum compound (III) is also confirmed by our experiments in which the copolymerization at as higher molar ratio of $[\mathrm{MA}]_{0} /[\mathrm{al}]$ as 25 was successfully carried out up to more than $90 \%$ conversion of methyl acrylate.

It was found that the copolymerization rate was promoted in the presence of peroxides such as benzoyl peroxide (BPO). The effect of benzoyl peroxide on the reaction rate of the copolymerization is shown in Figure 4. It was confirmed that in all cases an alternating copolymer of St-MA was obtained regardless 


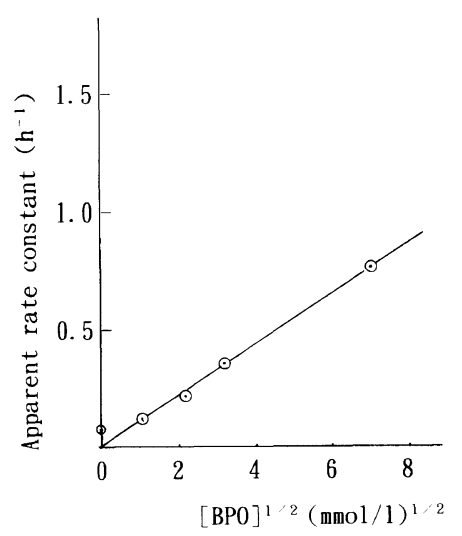

Figure 4. Effect of BPO concentration on the rate of copolymerization at $25^{\circ} \mathrm{C}$. Solvent, toluene; $[\mathrm{MA}]_{0}=0.4$ $\mathrm{moll}^{-1} ;[\mathrm{St}]_{0}=0.6 \mathrm{moll}^{-1} ;\left[\mathrm{AlEt}_{1.5} \mathrm{Cl}_{1.5}\right]=0.1 \mathrm{moll}^{-1}$.

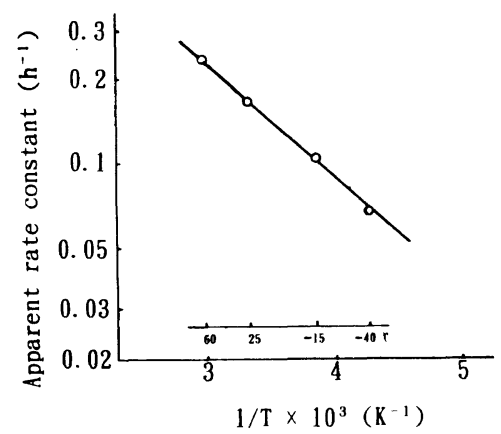

Figure 5. Effect of temperature on copolymerization of styrene and methyl acrylate. Solvent, toluene; $[\mathrm{MA}]_{0}=0.4$ $\mathrm{moll}^{-1} ;[\mathrm{St}]_{0}=0.6 \mathrm{moll}^{-1} ;\left[\mathrm{AlEt}_{1.5} \mathrm{Cl}_{1.5}\right]=0.2 \mathrm{moll}^{-1}$.

of peroxide concentration. The relation that the rate of the copolymerization is proportional to 0.5 power of [BPO] would be predicted from the eq 8 because [BPO] concentration might be involved in $R_{\mathrm{i}}$ of the eq 8 . This result would also support the validity of the above-mentioned kinetic assumptions.

The promoting effect was also observed with various kinds of peroxides. Lauroyl peroxide
(LPO) and BPO which have relatively large decomposition rate constants showed remarkable promoting effect. But the quantitative interpretation on the promoting effect of various peroxides will be discussed with further study.

The activation energy of the copolymerization was caluculated based on the data shown in Figure 5. The value is $1.82 \mathrm{kcal} \mathrm{mol}^{-1}$ which is substantially lower than that of the conventional radical polymerizations. ${ }^{11}$ This indicates that the activation energy of the propagation would decrease remarkably by forming a complex.

The proposed explanation and speculations might be firmly verified by further study conducted from various aspect of investigations.

\section{REFERENCES}

1. J. M.Cowie, Comprehensive Polym. Sci., 4, 377 (1989).

2. M. Hirooka, H. Yabuuchi, S. Morita, S. Kawasumi, and K. Nakaguchi, J. Polym. Sci., B, 5, 47 (1967).

3. M. Hirooka, H. Yabuuchi, J. Iseki, and Y. Nakai, J. Polym. Sci., A-1, 6, 1381 (1968).

4. M. Hirooka, H. Yabuuchi, S. Kawasumi, and K. Nakaguchi, J. Polym. Sci., Polym. Chem. Ed., 11, 1281 (1973).

5. C. H. Bamford, in "Alternating Copolymers," Ed. J. M. Cowie, Plenum, New York N.Y., 1985.

6. M. Hirooka, Pure Appl. Chem., 53, 681 (1981).

7. J. Barton and E. Borsig, "Complexes in Free-radical Polymerization” Elsevier, New York, N.Y., 1988.

8. J. Furukawa, E. Kobayashi, S. Nagata, and T. Moritani, J. Polym. Sci., Polym. Chem. Ed., 12, 1799 (1974).

9. H. Hirai and H. Komiyama, J. Polym. Sci., Polym. Chem. Ed., 12, 2701 (1974).

10. Y. Komo, S.Kondo, and K. Iimura, J. Polym. Sci., Polym. Lett. Ed., 12, 153 (1974).

11. T. F. McKenna and A. E. Hamielec, in "Polymer Handbook," 3rd ed, J. Brandrup and E. H. Immergut, Ed., Wiley Interscience, New York, N.Y., 1989, p II/335. 